\title{
CAMBIOS EN EL SISTEMA UNIVERSITARIO CHILENO: REFLEXIONES SOBRE SU EVOLUCIÓN Y UNA PROPUESTA DE GOBERNANZA
}

\author{
Juan Antonio Rock, Claudio Rojas ${ }^{1}$
}

\section{RESUMEN}

El ensayo discute una propuesta de gobernanza en las universidades públicas (estatales) chilenas. Toma como base las tendencias que afectan al sistema universitario mundial: masificación, diversificación, apertura a nuevos grupos de interés (stakeholders) y su correspondencia en el sistema universitario nacional. En el análisis se distinguen tres niveles: meta, contexto de valores y concepciones filosóficas, sociales, económicas, que conducen el concepto y las expectativas sobre la universidad. Un nivel meso, institucional, que involucra la estructura del aparato público y de gobierno responsable del sistema universitario, y un nivel micro, de las instituciones de educación universitarias, en el que son relevantes las estructuras de gobierno universitario, su gobernanza, liderazgo y gestión institucional.

Palabras clave: universidad, gobernanza, sistema universitario, políticas públicas

\section{CHANGES IN THE CHILEAN UNIVERSITY SYSTEM: REFLECTIONS ABOUT ITS EVOLUTION AND A PROPOSAL OF GOVERNANCE}

\section{ABSTRACT}

This essay discusses a proposal of governance for Chilean public universities (State). It is based on trends affecting the university system all over the world: overcrowding, diversification, opening to new stakeholders and its correspondence in the national university system. This analysis distinguishes three levels: a meta level, meaning value context and economic, social and philosophical ideas leading to the concept and expectations about the university; a meso level - the institutions - involving the government and the public structure responsible for the university system; and a micro level, university education institutions, where there are remarkable factors as university government structures, their governance, leadership and institution management.

Keywords: university, governance, university system, public policies

1 Académicos Universidad de Talca. Talca. Chile. Contacto: clrojas@talca.cl 


\section{CAMBIOS EN EL SISTEMA UNIVERSITARIO CHILENO: REFLEXIONES SOBRE SU EVOLUCIÓN Y UNA PROPUESTA DE GOBERNANZA}

Una reflexión sobre el sistema universitario chileno y sus requerimientos de cambios debe partir examinando la evolución del concepto de universidad como institución, su misión, valores, rol y estructura, y cómo estas se han ido plasmando en la sociedad contemporánea.

Distinguiremos en el análisis tres niveles ${ }^{2}$ : meta, es decir, el contexto de valores, y concepciones filosóficas, sociales, económicas que conducen el concepto y las expectativas sobre la universidad. Meso, que involucra la estructura del aparato público y de gobierno responsable del sistema universitario, la normativa legal, y las políticas y estrategias públicas; y micro, de las instituciones de educación universitarias, en el que son relevantes, las estructuras de gobierno universitario, su gobernanza, liderazgo y gestión institucional. Los dos primeros niveles se discuten a la luz de la experiencia global y nacional. El tercero, microinstitucional, se plantea como un ámbito de diagnóstico y propuesta para el sistema de universidades estatales chilenas respecto de las dimensiones argumentadas en los niveles meta y meso del conjunto universitario nacional.

\section{Concepción, misión y objetivos de la universidad}

Entendemos la universidad como el espacio institucional que se da una sociedad para el cultivo y generación del conocimiento y las artes y su transmisión, así como para el desarrollo de competencias profesionales, todo ello basado en el diálogo racional como método, el pluralismo y el rigor en las prácticas académicas. Es una definición extensamente difundida que compartimos.

2 Distinciones efectuadas a partir de Esser, Hillebrand, Messner y Meyer-Stamer (1996). 
Más ampliamente aún, entendemos la universidad como un constructo social para la transmisión de la cultura (Ortega y Gasset, 1930), considerando a esta como el sistema vital de ideas imperante en cada tiempo sobre el mundo, que permite superar el desorden y la entropía al que se vería enfrentado el hombre sin este contexto vital en sus interacciones con sus semejantes. Es la cultura de cada época y lugar la que influye en los métodos y valores que conducen la generación y transmisión de conocimientos y la formación de graduados y profesionales, de la que se hace cargo la universidad como institución.

La cultura evoluciona y se desarrolla. De acuerdo a su proceso histórico y territorial deviene en un sistema vital de ideas predominantes, que determina cómo los hombres interactúan, se ocupan de los asuntos públicos y privados, y de las cuales la universidad ha constituido uno de sus vehículos predominantes.

McCaffery (2010) sintetiza la evolución de este concepto de universidad portadora de un sistema vital de ideas y de cómo estas se plasman históricamente. Destaca la visión escolástica del cardenal Newman en el siglo XIX, enraizada desde la cultura griega clásica, quien concibe como principal misión de la universidad perseguir el conocimiento por sí mismo, el conocimiento liberal, sin utilitarismo alguno, que permita cultivar en los estudiantes los valores de una reflexión civilizada, no sujeta a compromisos con el Estado, los negocios, e incluso la Iglesia. La concepción de Wilhelm von Humboldt prioriza la investigación en la ciencia y la filosofía de los estudiantes graduados por sobre la enseñanza de pregrado, bajo la más plena libertad académica de profesores y estudiantes. La de Karl Jaspers considera a la universidad como una comunidad de eruditos comprometidos en la tarea de buscar la verdad, la que, sin embargo, no excluye la educación profesional, y la concepción napoleónica concibe a la universidad al servicio de los intereses de la nación por medio de la formación de profesionales y técnicos.

Ya en los inicios del siglo XX surge la concepción de la universidad "moderna", que combina las ideas de Newman y Von Humboldt, en búsqueda de una excelencia que permita superar lo que 
él califica como calidad mediocre de la investigación y la enseñanza de su época. En la segunda mitad del siglo el concepto de multiversidad planteado por Kerr (2007), en el que concibe a la universidad como la ciudad del intelecto, que responde a las demandas de diferentes grupos de interés o comunidades, académicos, ex alumnos, gobierno, negocios y otras instancias externas. En este último, académicos y estudiantes se identifican con subculturas específicas más que con una única cultura.

Finalmente, a las anteriores, debemos agregar la universidad global emergente de Mohrman, Ma y Baker (2008), generada por la aceleración de la globalización. Una universidad que exhibiría ocho características: misión global, intensa investigación, nuevos roles para los profesores, financiamiento diversificado, reclutamiento en todo el mundo, creciente complejidad, nuevas relaciones con el gobierno y la industria, y colaboración global con las instituciones pares. En consecuencia, la nación-estado de la cual es hija la universidad como institución moderna tiene cada vez menos influencia sobre sus instituciones globales de investigación, porque su trabajo se realiza más allá de los márgenes del estado-nación, el espacio privilegiado de regulación del pasado siglo XX.

Por otra parte, Altbach (2008) en una perspectiva histórica más amplia pero también preocupado por las funciones complejas que debe cumplir la universidad en la era actual de la globalización, señala que estas, además de preservar y difundir el conocimiento, deben ser centros intelectuales, es decir, instituciones en cuyo interior se debate y se proyecta el devenir social, para lo cual la libertad académica y la conciencia crítica son esenciales. Del mismo modo, advierte que cuando históricamente estas se dejaron de involucrar en la vida social y política, solían entrar en crisis, como fue el caso de la mayoría de las universidades europeas que no se involucraron en la Ilustración.

En síntesis, la universidad, como institución, muestra una evolución donde se la reconoce en su esencia como generadora de conocimiento y su transmisión. Funciones que se han ido paulatinamente complejizando mediante el reconocimiento de una creciente diversidad de interlocutores, cuyas demandas amplían y 
validan su misión. Inicialmente, la Iglesia y el Estado fueron sus principales sostenedores, posteriormente aparecen amplios grupos de interés (stakeholders), producto de la mayor complejidad que conllevan las interacciones sociales y económicas de la universidad en la globalización y la sociedad del conocimiento.

Actualmente en el mundo hay alrededor de 17 mil instituciones de educación superior (Hazelkorn, 2008), y la mayoría de ellas están principalmente enfocadas en la formación profesional, realizando alguna actividad de investigación en campos seleccionados. Las universidades de investigación, aquellas que privilegian la función generadora de conocimiento, rectoras actuales del sistema en su globalidad, representan una pequeña proporción del conjunto, pero son muy influyentes dentro del sistema de educación superior mundial (Mohrman et al., 2008). En Estados Unidos son 150 instituciones de un total de 7.000 instituciones de educación superior (McCaffery, 2010), no obstante tienen una influencia indudable en el sistema universitario norteamericano y mundial.

Altbach (2007) es enfático en destacar la necesidad de que los países en desarrollo focalicen sus capacidades en algunas universidades de investigación, sea por la necesidad de construir redes y eslabones con la construcción global del conocimiento, y también porque sus esfuerzos tienen que estar, en alguna medida, conectados con los requerimientos territorializados debido a que globalmente estos pueden no tener prioridad en las universidades líderes de la sociedad del conocimiento.

En general, se observa una creciente diversificación de las instituciones, tanto horizontal como vertical (Teichler, 2008), que obedece a una segmentación de los diferentes demandantes del sistema de educación superior. Como diversificación horizontal, han surgido las universidades docentes, de artes liberales, técnico-vocacionales, empresariales, de aprendizaje durante la vida y virtuales. En términos de diversificación vertical, se observan las referidas universidades de investigación (McCaffery, 2010).

Entre los desafíos contemporáneos que enfrentan las universidades, derivados de los requerimientos de cada país inducidos 
por el proceso de globalización y desarrollo de la sociedad del conocimiento, están: masificación y equidad; mejoramiento de la calidad de la formación; mayor involucramiento en el desarrollo económico y social; conciliar las tensiones entre el "colegialismo" tradicional y el emergente "gestionalismo" (Altbach, 2008); y atender los requerimientos de los diversos grupos de interés (stakeholders), preocupados por el desempeño de las instituciones.

En su conjunto, son desafíos que alteran o exigen redefiniciones en los niveles mesoinstitucionales universitarios: las políticas públicas que conducen sus estructuras y ordenamientos, sus contextos legales y de financiamiento, así como en un nivel micro; las estructuras de gobernanza, gestión y liderazgo institucional.

\section{Desafíos de las universidades con su entorno}

En un nivel de análisis mesoinstitucional, las universidades no son solo proveedoras, sino también dinamizadoras del entorno. No solo objeto de las políticas públicas, sino también sujetos. Tienen una oferta que crean a partir de sus propias realidades y trayectorias (Becher, 2001). Asimismo, como señalan Muga y Sotomayor (2010), salvo en las instituciones que tienen la capacidad de ejercer un liderazgo global, en las universidades "territorializadas" sus posibilidades de posicionamiento se vinculan con las oportunidades que les otorga el entorno, y las restricciones vienen más bien dadas de sus condiciones internas. Son importantes factores tales como redes, relaciones "cara a cara", institucionalidad de apoyo y oportunidades de desarrollo académico, que otorgan un ambiente local de gran atracción (Boisier, 2006; Rojas, 2001).

En la perspectiva de la universidad como actor del desarrollo son sugerentes las propuestas de Salmi (2009), Salmi y Altbach (2011) y Clark (2000). Clark se ha preocupado de caracterizar a las universidades emprendedoras y transformadoras en instituciones de tamaño intermedio en el contexto europeo. Los factores que identifica como relevantes son la calidad de dirección, la amplitud y calidad de la "periferia" (alianzas con la industria, creación de propiedad intelectual, spin-off, transferencia científico-tecnológica), 
financiamiento y diversificación, núcleo académico motivado, cultura emprendedora.

Con una mirada más amplia, Salmi señala los factores que discriminan que una universidad se convierta en una institución de excelencia y de clase mundial, como son la calidad de su gestión, los recursos que dispone, y su capacidad de atracción de talentos. Son factores que incidirían efectivamente en el progreso de universidades "emergentes" o de calidad intermedia, para lograr altos estándares y posicionamiento (Salmi y Altbach, 2011).

Al reconocer que estos factores no pueden ser trasladados mecánicamente, las referencias previas muestran que la relación con el entorno los afecta sustancialmente y determina el desempeño de las universidades. Además, este vínculo releva los marcos y diseños institucionales que estimulan las capacidades de aprendizaje y la difusión de innovaciones tecnológicas, que buscan que las empresas y el sistema económico en general transiten hacia fases productivas con mayor valor agregado, y donde los agentes e instituciones que proveen ciencia y tecnología son objeto de las políticas públicas.

\section{Rol de la política pública}

El diseño e implementación de las políticas públicas ha sido siempre la búsqueda del progreso y desarrollo nacional, y una de las dimensiones en que estas han avanzado es en la actuación de las instituciones de educación superior, especialmente el caso de las universidades. Como se ha dicho, no se asume en ellas una mirada solamente instrumental -objeto de políticas- sino que también se las reconoce como sujeto en ellas (Muga y Sotomayor, 2010).

En un nivel de precisión amplio, es necesario señalar que el papel del Estado no es solo el de aparato de control burocrático que asegure estabilidad y gobernabilidad. Su rol esencial -en un contexto de regulación democrática- es constituir el espacio donde la sociedad manifiesta sus expectativas del país que quiere vivir, de los valores que rijan la convivencia y los intercambios. Por eso que es legítimo hablar de política pública para el sistema de educación superior y 
las universidades públicas. Que estas no tengan un comportamiento autorreferido, y que respetando su identidad encaucen su desarrollo con los énfasis que el país desea.

En esta medida, la noción de "bien público" en el sistema universitario radica en que muchas de las acciones que realiza constituyen actividades complejas -culturales, sociales, formación de capital humano avanzado, investigación- (Altbach, 2008), que contribuyen a la formación profesional y técnica, así como a incrementar la capacidad de innovación y su difusión, como factor propulsor de la competitividad y de un régimen de crecimiento (acumulación) con equidad.

Las insuficiencias que eventualmente se observan en la generación de estos bienes públicos provienen tanto de fallas de mercado como de Estado. Entre las primeras, la insuficiente apropiación que conlleva la producción de estos bienes, la limitada información en el contexto que se da la educación superior emergente, sobre todo por las tasas de crecimiento que ha tenido el sistema de educación terciaria en el mundo. Entre los segundos, los problemas de coordinación que exhibe la política pública y las capturas corporativas que suelen experimentar las instituciones públicas. Ambos tipos de fallas, de mercado y de Estado, generan fuertes costos de transacción que inhiben la generación de conocimiento avanzado y desarrollo tecnológico, así como la gobernabilidad y alineamiento del sistema, y en cambio estimulan eventuales riesgos morales en el comportamiento de las diversas instituciones y sus miembros.

Una premisa en la que se basa este ensayo, es que habiendo políticas públicas en el ámbito del sistema universitario chileno, el dónde y cómo se generan ellas constituirían espacios poco atravesados por la discusión política y social chilena, y por tanto históricamente poco visibles. Su perfil político y social es bajo con relación a otros requerimientos nacionales. Llama la atención la baja prioridad que tradicionalmente ha ocupado esta dimensión de la labor universitaria en el debate político-social chileno, que más allá de ser reconocida como un vehículo de movilidad social, no se le ve como un canal privilegiado para apalancar el desarrollo. 
Los trabajos de Salmi (2009), Salmi y Altbach (2011), Kerr (2001, 2007) son solo algunos referentes de cómo en China (Hong Kong incluido), Singapur, India, California (EE.UU.), países con alta y baja cobertura universitaria, muestran una elevada sensibilidad del Estado y de la sociedad respecto del rol que sine qua non cumplen las instituciones académicas para enfrentar los desafíos económicos y sociales, mediante una educación superior de calidad y altos estándares de investigación y progreso tecnológico.

\section{Las estructuras de gobernanza y gobiernos corporativos de las universidades del Estado chileno: diagnóstico y propuesta}

Niveles meta y mesoinstitucionales: características y dimensiones emergentes del sistema universitario chileno

La sociedad chilena no ha estado ausente a los cambios culturales que afectan a la sociedad global, expresados, entre otros, en la masificación del sistema de educación superior, la aparición de nuevos grupos de interés, la manifestación y exigencia de la universidad como centro intelectual y de apoyo al desarrollo productivo. Es por ello que en sus niveles meta y mesoinstitucionales el sistema de educación superior chileno atraviesa un conjunto de dilemas y tensiones que no son ajenos a los descritos en general, aunque las particularidades nacionales le otorgan rasgos distintivos, que están presentes como ejes problemáticos para las políticas públicas y la conducción de las instituciones.

Se puede distinguir sucintamente, en primer lugar, un desequilibrio creciente entre las demandas que enfrenta el sistema universitario nacional y las respuestas que ofrece en investigación y posgrado; en formación profesional, mayor cobertura, equidad en el acceso y formación de calidad; en vinculación con el medio, educación continua e interacciones con el sector productivo y social. Constituyen un conjunto de demandas y requerimientos que no siempre encuentran respuesta oportuna en el sistema universitario chileno (Consejo Nacional de Innovación, 2008). 
En segundo término, un crecimiento acelerado e inorgánico del sistema universitario, basado en buena parte en el crecimiento de las universidades privadas a partir de la reforma de 1981. En 1985 había en el país 11 instituciones ( 2 estatales, 6 privadas de carácter "público", y 3 privadas sin financiamiento estatal directo). En 2008 contaba con 61 universidades; 16 estatales, 9 privadas "dependientes" y 36 privadas independientes. Entre 1985 y 2009 la población universitaria subió de 118.077 a 546.208 estudiantes, un 362,6\% de aumento, en tanto que la población del país en el mismo periodo creció solo un $39,9 \%$. En síntesis, un crecimiento que ha llevado progresivamente desde la formación de la élite a uno de carácter universal (Trow, 2000).

Este elevado incremento ha producido heterogeneidad creciente en el sistema universitario, generando tensiones tanto internas como interinstitucionales, afectando los mecanismos de coordinación del sistema en su conjunto. Del mismo modo, predominan estructuras de gobiernos corporativos preferentemente "colegiales", que generarían dificultades para relacionarse con el medio y sus diversos grupos de interés (Altbach y Salmi, 2011; Reyes, 2011), así como dificultades de control administrativo ex-ante en el caso de las universidades públicas, que le restarían flexibilidad de gestión y conducción estratégica. ${ }^{3}$

Tercero, estas heterogeneidades se manifiestan en las distintas dimensiones que atraviesan las instituciones, sea por sus capacidades de gestión y estructuras de gobierno diversas, por sus distintas capacidades de producir conocimiento (básico y aplicado), por diferentes talentos de los alumnos enrolados, por una distribución presupuestaria per cápita desigual, y un dispar grado de diversificación en las fuentes de financiamiento.

En esta perspectiva, y a pesar de los esfuerzos efectuados por los sucesivos gobiernos nacionales, existe bastante consenso respecto de la necesidad de mejorar sustancialmente la movilidad y articulación,

3 Al respecto ver Bernasconi (2011) donde analiza el caso específico de las universidades de Chile y Pontificia Católica de Chile. Junto con ello, es interesante destacar los resultados preliminares de Rojas (2012) donde a partir de los datos de panel se muestra una significativa mayor capacidad de vinculación con el medio -medida en venta de bienes y servicios y transferencia tecnológica- de las universidades privadas del $\mathrm{CRUCH}$, respecto a las instituciones estatales de esta. 
tanto en el pregrado como en el posgrado, entre la formación técnica y profesional, así como también en la necesidad de superar las brechas en las capacidades de investigación, desarrollo tecnológico e innovación, y en la formación de capital humano avanzado (Cáceres, 2010, Bernasconi, 2011). Así también, de no persistir en una suerte de uniformidad en las políticas, que no da cuenta cabal de las diferentes misiones y propósitos institucionales (entidades estatales, privadas, confesionales, metropolitanas, regionales), y de sus diferentes trayectorias de aprendizaje (Lemaitre, 2009).

Por otra parte, también es notable el bajo perfil, tanto político y social, que ocupan las políticas públicas y recursos destinados a I+D+i (investigación, desarrollo tecnológico e innovación) más allá del reconocido papel como vehículo de movilidad social que se le atribuye a la formación superior universitaria chilena. A pesar del alto gasto nacional en educación superior, 2,1\% del PIB, el aporte público es muy bajo, no superior al 0,7\% del PIB, considerablemente inferior al promedio de los países de la OCDE (OECD, 2007). Así también el gasto en investigación, desarrollo tecnológico e innovación (I+D+i), no es mayor al 0,4\% del PIB (CRUCH, 2009).

En general, el sistema nacional refleja la masificación y desregulación que señala la tendencia mundial, y una apertura al medio -principalmente en lo que respecta a las fuentes de financiamiento, menos en lo que se refiere a las necesidades de desarrollo económico y social- que está creando mayor complejidad y diversidad en los sistemas académicos. Oportunidades de acceso sin precedentes pero que al mismo tiempo crea sistemas que son menos igualitarios y más difíciles de financiar (Altbach, 2008; Lavados, 2006).

Se ha tensionado también el clima de conducción de las universidades, producto de la emergencia del "gestionalismo", resultado de las necesidades de administración que enfrentan las instituciones y que muchas veces no dialoga bien con el "colegialismo" tradicional de las instituciones de educación superior (Brunner, 2010; Altbach, 2008).

En otros términos, la gobernabilidad de las instituciones públicas ha pasado a constituir una cuestión crítica en el sistema 
(Marshall, 2010; Cáceres; 2010). De acuerdo a Brunner (2010), el sistema universitario ha transitado a un escenario en que las condiciones en que se desenvuelven las universidades son de una profunda "mercadización y privatización", con una disciplina que en buena parte la impone el mercado. Un escenario que desafía la política pública en el desarrollo de condiciones de gobernabilidad, especialmente en las universidades estatales, que estimula en las instituciones una "alineación" en función de propósitos de interés nacional.

Un aspecto crítico es cómo superar una baja capacidad de incentivar el desempeño de las universidades de "bien público" (como también se denomina a las instituciones del Consejo de Rectores de la Universidades Chilenas, CRUCH) en función de objetivos de desarrollo nacional. En esto inciden la referida "mercadización" del sistema, pero también las estructuras de gobierno corporativas y los sistemas de incentivos que reportan los sistemas de financiamiento existentes $^{4}$. Esto no significa señalar que lo que ha dominado la escena es una ausencia del Estado chileno en la conducción del sistema universitario nacional. Por cierto, ha habido una presencia importante de instrumentos públicos de financiamiento, tanto para la oferta como para la demanda, y que han logrado estimular la expansión del sistema, levantando nuevas capacidades, en sistemas de información y gestión institucional, y rediseño curricular, entre las más connotadas (Lemaitre, 2005), pero sus alcances y velocidades de implementación se han visto limitadas por las capacidades de "inicio" de las diversas instituciones (Cáceres, 2010; Rojas, 2005).

En consecuencia, lo que correspondería es un rediseño de las políticas públicas relativas al acceso y equidad del sistema, promoción de la investigación básica y aplicada, un perfeccionamiento de los mecanismos de rendición de cuentas por los recursos asignados. Del mismo modo, se requerirían reformas en las estructuras de gobernanza y gestión de las universidades, y en la misma institucionalidad estatal (Marshall, 2010; Cáceres, 2010), de forma que el desempeño

4 Que está basado en una productividad académica derivada de la generación de conocimiento avanzado más que en investigación aplicada y de transferencia tecnológica. 
de las instituciones universitarias, tanto públicas como privadas, se estimulen a atender los requerimientos del desarrollo nacional. Asimismo, se requiere una mayor articulación del sistema (Lavados, 2006) como también profundizar y perfeccionar los procesos de acreditación.

En síntesis, en este nivel meta y mesoinstitucional se puede señalar que el sistema universitario nacional vive: 1. Una masificación acelerada en un contexto de "mercadización" y creciente heterogeneidad en el sistema de las universidades estatales, tanto en capacidades de gestión como en estructuras de financiamiento. 2. Un déficit de "agencia" y falta de claridad en el rol del Estado, que afecta el direccionamiento estratégico de las universidades, especialmente las estatales, en su relación con el medio (Reyes, 2011, Bernasconi, 2011). 3. Un predominio en el sistema de estructuras de gobiernos corporativos preferentemente "colegiales" que generarían dificultades para relacionarse con el medio y sus diversos grupos de interés (Altbach y Salmi, 2011; Reyes, 2011).

\section{Nivel microinstitucional}

En el nivel microinstitucional, las universidades públicas, por su naturaleza, debieran disponer de autonomía y libertad en su desempeño para cumplir con sus respectivas misiones y objetivos, respetando las disposiciones legales del país. Asimismo, debieran practicar los valores de transparencia y responsabilidad social respecto de sus promesas de misión y objetivos, informando todo su quehacer académico a la sociedad. No obstante ello, corresponde al Estado establecer una institucionalidad con capacidad para expresar y conducir demandas sociales por medio del sistema de educación superior, regulando, informando y controlando su cumplimiento.

Las instituciones privadas, respetando la legislación vigente, tienen la opción, en uso de su autonomía y libertad académica, de conducir la práctica de algunos valores universitarios relativos al pluralismo ideológico, en función de sus principios y concepciones ideológicas. Del mismo modo, las instituciones privadas pueden definir la gobernanza que les sea más apropiada. Por su parte, las 
instituciones del Estado deberían practicar ampliamente los valores de pluralismo ideológico, integración social, vocación pública y bien común, solidaridad y promoción de los valores democráticos, además de los propios del quehacer universitario.

\section{Fines, estructura y gobierno de las universidades del Estado}

Como se ha señalado, las universidades del Estado deben necesariamente contribuir a crear las condiciones para el desarrollo del territorio en las que están insertas y apoyar a las personas y la sociedad para incrementar sus opciones y oportunidades de destino. De igual forma, la estimulación de la ciudadanía para modelar una sociedad inclusiva y democrática es una dimensión importante del compromiso con el bien común de las universidades estatales, el que por cierto no excluye a otras instituciones de educación superior que así lo definan.

Un desafío que es vital para la universidad y su servicio a la sociedad, es conciliar la búsqueda de la verdad, el debate, la práctica del pensamiento crítico frente al poder de los diversos grupos de interés, del Gobierno o de quien financia o administra la institución. Es el reto de la universidad en su esencia, pero que debe conciliarse con las demandas de apertura a distintas comunidades de intereses, que también debe sujetarse -en la estructuración del gobierno universitario- a un balance entre el poder de la administración y la autoridad de los académicos. Ello solo será posible, como lo señala Sporn, "si ambos grupos rinden cuentas basados en objetivos medibles y aceptados" (Sporn, 2009, p. 237). Esto permite reconciliar un modelo de gobierno compartido, pero de necesaria ejecutividad y flexibilidad, de "colegialidad" y "gestionalismo" como señaláramos anteriormente.

En la actualidad la universidad debe servir a muchas comunidades o grupos de interés, lo que ha sido muy bien expresado en el concepto de multiversidad de Kerr (2001). En el plano interno, a estudiantes, académicos, y funcionarios administrativos. En el entorno, a exalumnos, Gobierno, empresas, y comunidades locales. 
No obstante, existe la necesidad de darle gobernanza a las instituciones universitarias en función de los propósitos de sus grupos de interés legítimos, vale decir, liderazgos y estructuras de gestión y gobierno coherentes con el conjunto de estas comunidades. Esto obviamente involucra al poder..."la gobernanza concierne al poder. Es acerca de quién está encargado; quién toma las decisiones; quién tiene voz; y cuán fuerte suena esa voz". ${ }^{5}$ Suponemos que los distintos grupos legítimos de interés de las universidades públicas deben tener la oportunidad de hacer oír su voz.

\section{Estructura de gobierno de las universidades estatales chilenas. A modo de evaluación y propuesta de gobernanza}

De acuerdo a consideraciones anteriores, lo que observamos son estructuras de gobierno de las universidades estatales de Chile que han cumplido 30 años de vigencia. Existe por tanto una experiencia acumulada que permite un análisis de los factores más críticos que afectan dicha estructura.

1. La mayoría de las instituciones tienen estatutos comunes que estandarizan sus estructuras, sin considerar las diferencias de tamaño de cada una, lo que a muchas de ellas les significa soportar estructuras inadecuadas y costosas.

En 1981 se reestructuraron las universidades del Estado; la De Chile y la Técnica del Estado, dando origen a la Universidad de Santiago y a las universidades derivadas de ambas. Los estatutos que han regido al conjunto de estas contemplaron un gobierno y estructura similar, con excepción de la Universidad de Chile, que posteriormente por ley modificó su estatuto, y las universidades De Los Lagos y Tecnológica Metropolitana, ambas creadas con posterioridad a 1981 .

En los estatutos estándares que rigen a la mayoría de las universidades estatales (OECD, 2007), la más alta instancia de gobierno es la junta directiva, integrada por un igual número de

5 Henry Rosovsky (1990), decano de Harvard, citado en McCaffery (2010). 
representantes del Presidente de la República; miembros externos, nombrados por el consejo académico, y representantes de los académicos nombrados por el consejo académico (en algunos casos a instancias del cuerpo académico).

Los estudiantes y personal administrativo carecen de participación en el gobierno universitario, con la excepción de las universidades de Chile, de Los Lagos y Tecnológica Metropolitana, que consultan su participación en sus cuerpos colegiados (OECD, 2007).

Las características del gobierno universitario y su estructura descritas se replican igualmente en una universidad compleja del tamaño de la de Santiago con 19.000 estudiantes de pregrado como en una universidad de menor complejidad y tamaño como la de Magallanes con 2.830 estudiantes.

2. Las juntas directivas tienen una composición mayoritariamente no académica, puesto que dos tercios de sus integrantes son externos a la universidad, pero paradojalmente tienen atribuciones en términos de aprobación de programas, planes de estudios, y certificaciones académicas, lo que no guarda relación con la naturaleza de su composición. Esto, además de la discordancia señalada puede, potencialmente, llegar a generar tensión con la autonomía, colegialidad y libertad académica.

3. Tampoco es coherente que en el directorio no exista participación de grupos de interés específicos (representantes de la sociedad civil o de los empleadores). Esto refuerza la dirección institucional y estimula la rendición de cuentas (OECD, 2007).

4. Los estatutos no contemplan participación de los estudiantes en los organismos colegiados de las universidades, lo que puede resultar inconveniente para el necesario equilibrio que deben tener para atender a sus diferentes comunidades internas. Sobre todo, si se considera la permanente tensión entre las dimensiones de formación de pregrado e investigación, formación de posgrado, innovación y transferencia tecnológica.

5. Los estatutos actuales tampoco contemplan participación de los funcionarios no académicos en las instancias colegiadas pertinentes, lo que es paradójico con las tendencias de la gestión moderna de las organizaciones.

6. El rector y los decanos son elegidos por sus pares académicos, los 
que posteriormente son sus subordinados, desde el punto de vista administrativo. Esta situación genera condiciones para responder en mayor medida a los intereses de electores académicos, que al resto de las comunidades interesadas, lo que afecta el cumplimiento cabal de la misión universitaria. La situación ideal es generar las condiciones para un equilibrio entre la necesaria colegialidad, libertad y autonomía universitaria y el servicio a las comunidades externas de las universidades.

7. Es necesario que la estructura de gobierno universitario proteja apropiadamente la autonomía y libertad académica de las universidades, sus valores históricos esenciales. Asimismo las universidades deben adoptar mecanismos de gestión adecuados para atender el crecimiento y la diversidad de demandas del medio externo, las que han diversificado sus fuentes de financiamiento y generado nuevos compromisos. Se trata de alcanzar un equilibrio entre dos tendencias: el "colegialismo" (colegialism) y "gestionalismo" (managerialism) (Deem, 2008; Mohrman et al., 2008; Altbach, 2007).

8. Por último, si bien las universidades estatales tienen libertad para establecer sus remuneraciones y carrera académica, los reglamentos de la administración pública que les competen hacen extremadamente difícil la gestión de recursos humanos y limitan la plena incorporación de académicos extranjeros (OECD, 2007). En el ámbito de la regulación administrativa, el control ex-ante de la Contraloría General de la República en materias relativas a inversiones, adquisiciones y nombramientos académicos resta flexibilidad en el accionar de las instituciones del Estado (OECD, 2007).

\section{Propuesta de gobernanza}

La estructura de gobierno será apropiada en la medida que sea funcional a la estrategia de la institución, sus procesos, cultura y características de magnitud. En el caso de las universidades del Estado de Chile, se observa una diversidad de instituciones: metropolitanas y regionales; docentes y de investigación; matrices y derivadas; grandes y pequeñas. En consecuencia, es conveniente definir solo 
los parámetros básicos de la estructura y dejar que cada institución termine por definir la globalidad de esta.

No obstante, teniendo en consideración las dimensiones de capacidad de interlocución con los diversos grupos de interés y de respuesta a las necesidades de desarrollo nacional, un conjunto de componentes básicos de la estructura serían el consejo directivo; el rector y su tecnoestructura; el senado y su consejo académico y la contraloría interna.

\section{El consejo directivo}

El Estado debe delegar en este consejo la gestión estratégica, presupuestaria y de inversiones; la aplicación de las políticas públicas de educación superior, la rendición de cuentas públicas y las relaciones internacionales de la universidad.

Sus consejeros deben ser nombrados por periodos largos, que trasciendan los presidenciales. Un periodo de 10 años, como el de los consejeros del Banco Central, parece bastante adecuado, y sin derecho a reelección. Su renovación debe parcializarse en el tiempo, con el objeto de evitar que su composición esté sesgada a una determinada corriente política, predominante circunstancialmente.

El consejo debiera tener doce miembros permanentes, responsables solidariamente de sus decisiones, cuyo ejercicio del cargo debiera ser remunerado. El Presidente de la República debiera nombrar cuatro consejeros; el gobierno regional dos; el senado académico seis -cuatro académicos y dos exalumnos-. Además de los miembros permanentes, debieran integrar el consejo un representante de los funcionarios no académicos y dos representantes de los estudiantes, elegidos por ambos estamentos.

\section{Senado académico}

El senado académico se debiera encargar de todos los asuntos académicos de la institución, por medio del cual los académicos ejercerían su plena autonomía y colegialidad. Entre las funciones académicas de mayor importancia cabe señalar: aprobar programas conducentes a 
grado, profesionales y de actualización y perfeccionamiento, y sus correspondientes planes de estudio; otorgar títulos y grados; dictar reglamentos académicos; aprobar políticas de admisión; aprobar políticas, procedimientos y reglamentos concernientes a la selección, evaluación, promoción, perfeccionamiento y remoción del personal académico.

El senado académico estaría constituido por profesores de la universidad con contratos hasta la edad de retiro, elegidos por los académicos, nombrados por periodos de tres años, pudiendo ser reelegidos. El número de sus miembros dependerá del número de académicos de la institución, el que no debería exceder a cuarenta y cinco. Se integrarían al senado, con plenos derechos, cinco representantes de los estudiantes, especialmente elegidos.

Debiera poder establecer comités permanentes, presididos por miembros del senado e integrado además por académicos que no pertenecen a este, para elaborar propuestas y estudios concernientes a materias académicas.

El senado de cada universidad determinaría la estructura organizacional de las actividades académicas, pudiendo crear facultades, departamentos, institutos y otras unidades que sean necesarias para relacionarse con los sectores productivos e institucionales externos.

El presidente del senado debe ser el rector y el vicepresidente, elegido por este, oficiaría de presidente de la asamblea en ausencia del presidente.

\section{Rector}

El rector debe ser la autoridad unipersonal de mayor jerarquía de la institución, y responsable de la gestión administrativa y académica de la corporación y de su representación jurídica en el medio externo. Debe tener la responsabilidad de preparar el plan estratégico y los presupuestos anuales de gasto e inversión que serán sometidos al consejo directivo, como también de su correspondiente ejecución. Asimismo, debe ser responsable de supervisar la correcta ejecución 
de las decisiones del senado académico.

Con el propósito, como ya se ha señalado, de que la institución responda adecuadamente a las demandas de la sociedad, es apropiado que el rector no solo represente a los académicos, sino también a todas las comunidades que tienen interés en la universidad. Para ello sería recomendable que el proceso de nombramiento del rector permita una participación más amplia que la actual.

\section{Contralor interno}

El contralor interno debería ser nominado por el consejo directivo por un periodo amplio (por ejemplo 10 años), y duraría en el cargo mientras cuente con la confianza de este.

El contralor debe ser responsable del control interno y legal, como también de la prevención y auditoría de riesgo. Debería elaborar informes periódicos dirigidos al consejo directivo, los que tendrían carácter público.

Finalmente, dentro de la institucionalidad del Estado, es interesante considerar la instalación de un consejo de educación superior, integrado por miembros de grupos de interés motivados en el progreso y mejoramiento permanente del sistema universitario, como una instancia de interlocución del Estado con sus universidades. Estas debieran tener el mismo tratamiento jurídico de las empresas autónomas del Estado, debiendo rendir cuentas ex-post ante la Contraloría General de la República. Las normas generales de administración del Estado les serían aplicables en todas las materias, en tanto no tengan normas correspondientes propias.

Nos asiste la convicción de que la estructura de gobernanza propuesta protege adecuadamente la autonomía, colegialidad y libertad académica, pero junto con ello genera condiciones para atender adecuadamente a las diversas comunidades externas, grupos de interés y estímulo y mejores conexiones con las demandas del medio. 


\section{Financiamiento}

Es preciso reconocer dos ámbitos: financiamiento a la demanda y financiamiento a la oferta. El primero se relaciona principalmente con los aranceles de pregrado y posgrado, y las becas y créditos institucionalizados que se otorguen. El criterio principal es que los aranceles deberían cubrir el costo de la docencia, incorporando becas ya sea para posibilitar el acceso de los grupos vulnerables que no los puedan costear, y/o por los beneficios sociales que signifiquen determinados programas.

El segundo tipo de financiamiento se vincula con los bienes públicos, más allá de la docencia, que provean las instituciones, es decir, extensión, investigación, desarrollo tecnológico e innovación. En este punto, se debieran reconocer esencialmente convenios de desempeño -para generar alineamiento con requerimientos de la sociedad y transparencia en su utilización- de acuerdo a criterios relacionados con la trayectoria y desafíos específicos de cada institución.

\section{Síntesis conclusiva}

Reconociendo las distinciones de análisis efectuadas en este ensayo, se destacan los siguientes aspectos resumidos:

1. En su esencia a la universidad se la reconoce como un instrumento de la sociedad para la transmisión de la cultura, mediante la generación de conocimiento, la formación de competencias profesionales y su difusión. Una función que se ha ido paulatinamente complejizando, por el escrutinio de una creciente diversidad de interlocutores cuyas demandas amplían y validan su misión.

En este nivel metainstitucional, el sistema nacional refleja la masificación que señala la tendencia mundial, y una apertura al medio, que está creando mayor complejidad y diversidad en los sistemas académicos. Es un crecimiento que ha amplificado las tensiones intraorganizacionales entre la docencia de pre y posgrado, entre la docencia y la investigación, entre la investigación básica y la investigación aplicada.

2. En el nivel mesoinstitucional, un desafío que enfrenta la política pública es el desarrollo de condiciones de gobernabilidad, 
especialmente en las universidades estatales, que estimulen un "alineamiento con propósitos nacionales" en las instituciones.

La noción de "bien público" en el sistema universitario radica en que muchas de las acciones que realiza constituyen actividades complejas -culturales, sociales, formación de capital humano avanzado, investigación- que contribuyen a una mayor competitividad nacional y crecimiento con equidad. Los problemas de coordinación que exhibe la política pública y las capturas corporativas que suelen sufrir las instituciones públicas, y las fallas de mercado y de Estado, generan costos de transacción que inhiben la generación de conocimiento avanzado e innovación, así como la gobernabilidad y alineamiento del sistema, y los eventuales riesgos morales en el comportamiento de las diversas instituciones y sus miembros.

3. En el nivel micro, las instituciones universitarias, especialmente las públicas, enfrentan dos escollos para responder a este requerimiento de alineamiento con los objetivos nacionales: 1) estructuras de incentivos y estructuras de gobierno en poca correspondencia con las demandas del medio, y 2) eventuales déficits de gestión y de financiamiento.

Las instituciones del Estado deben tender a practicar ampliamente los valores de pluralismo ideológico, integración social, vocación pública y bien común, solidaridad y promoción de los valores democráticos, además de los propios del quehacer universitario. La estructura de gobierno será apropiada en la medida que sea funcional a la estrategia de la institución, sus procesos, cultura y características de magnitud; así también esta debe ser coherente con la capacidad de dar respuesta a los requerimientos de sus diversos grupos de interés, incluidos los requerimientos de respuesta a las necesidades de desarrollo del país.

Considerando la diversidad de las universidades del Estado es conveniente solo definir los parámetros básicos de la estructura y dejar que cada institución termine por definir la globalidad de ella. Los componentes básicos de la estructura que se propone contemplan el consejo directivo; el rector y su tecnoestructura; el senado y su consejo académico y la contraloría interna. El criterio fundamental es que el consejo directivo -organismo colegiado 
principal encargado de su conducción estratégica- represente la diversidad de grupos de interés presentes (interesados) en el desempeño universitario, y un senado, que arbitre la conducción académica de la institución, donde estén representados los diversos grupos internos, aunque con mayoría del estamento académico.

4. El financiamiento de las universidades del Estado debe reconocer el ámbito de la demanda (aranceles) y de la oferta (generación de bienes públicos en extensión, investigación y programas profesionales de alta rentabilidad social). Debe distinguir las diversas circunstancias y etapas de desarrollo institucional, y contar con incentivos para una mayor interacción con el entorno socioproductivo.

5. Por último, existen interconexiones entre los diferentes niveles de análisis. Sin duda que los cambios culturales que vive la sociedad chilena - movilidad social, globalización-, la emergencia de nuevos grupos de interés, y las expectativas que se tienen del sistema universitario, todas dimensiones propias de un nivel metainstitucional, están afectando el nivel mesoinstitucional del sistema y en específico el contexto en el cuál se desenvuelven las instituciones.

En concreto, las principales dificultades que se observan es el alineamiento entre las expectativas y demandas que la sociedad plantea a las universidades, representada por el Estado, y la respuesta de las universidades. Es necesario precisar la identificación y alcance de las expectativas de la sociedad, por una parte, y diseñar los incentivos y apoyos adecuados del Estado que permitan atender dichas expectativas. Producir los consensos sociales requeridos constituye un desafío mayor.

A nivel meso, el principal desafío es el diseño de políticas públicas que permitan atender la producción de los bienes sociales de la educación superior en una perspectiva de largo plazo, pensando en la sociedad chilena del futuro que deseamos. Es la necesidad de una política pública que incorpore las complejidades de las funciones universitarias, dadas por diferentes trayectorias de aprendizaje de disímiles instituciones, sus particularidades territoriales y diversos grupos de interés que enfrentan. Es decir, apuntando a pensar y 
estimular los cambios sociales necesarios para generar amplias oportunidades de destino a los individuos, regiones y país.

En lo micro, el mayor desafío es superar el ensimismamiento de las instituciones como comunidades cerradas, en una concepción clásica obsoleta en la sociedad globalizada del conocimiento, para trascender a instituciones capaces de sintonizar con las aspiraciones de los diversos y legítimos grupos de interés (académicos, estudiantes, gobierno nacional y local, empresas, instituciones públicas, organizaciones sociales y culturales). Este desafío requiere que las instituciones, además de responder al concepto de comunidad, también sientan como parte de su legitimidad el servicio a fines externos a ellas, para lo que deben dotarse de sistemas de gobierno y capacidades de gestión adecuadas.

\section{Referencias bibliográficas}

Altbach, Ph. y Salmi, J. (2011). The Road to Academic Excellence: Emerging Research Universities in Developing and Transition Countries (manuscript).

Altbach, Ph. (2007). Peripheries and Centres: Research universities in developing countries. Higher Education Management \& Policy, 19, 111-134.

Altbach, Ph. (2008). "Funciones complejas de las universidades en la era de la globalización”, en La Educación Superior del Mundo, Ed. Mundi Prensa.

Becher, T. (2001). Tribus y Territorios Académicos: La Indagación Intelectual y las Culturas de las disciplinas, GEDISA.

Bernasconi, A. (2011). "Private and public pathways to world-class research universities: The case of Chile”, en Altbach, Ph. y Salmi, J. (Eds.) The Road to Academic Excellence: Emerging Research Universities in Developing and Transitions Countries, Banco Mundial (en impresión).

Bleiklie, I. y Kogan, M. (2007). Organization and Governance of Universities. Higher Education Policy, 20, 477-494.

Boisier, S. (2006): Imágenes en el Espejo: Aportes a la Discusión sobre Crecimiento y Desarrollo Territorial, en Ed. Puerto de Palos.

Brunner, J.J. (2009). Educación Superior en Chile, Instituciones, Mercados y Políticas Gubernamentales, Santiago: Ed. Universidad Diego Portales. 
Cáceres, C. (2010). Financiamiento, institucionalidad y gestión en las universidades: Desafíos y propuestas, Estudios Sociales n. ${ }^{\circ} 118$.

Clark, B. (2000). Crear Universidad Emprendedora: Trayectorias Organizativas hacia la Transformación, OCDE, Conferencia General IMHD, París.

Consejo Nacional de Innovación (2008). Hacia una Estrategia Nacional de Innovación para la Competitividad, vol. I y II.

CRUCH (2009). El Consejo de Rectores ante los desafíos de las universidades chilenas en el Bicentenario. Santiago. CRUCH.

Davies, J., Douglas, A. y Douglas, J.A. (2007). The effect of academic culture on the implementation of the EFQM Excellence Model in UK universities. Quality Assurance in Education, 15, 382-403.

Deem, R. (2008). Producing and re/producing the global university in the 21 st century: researcher perspectives and policy consequences. Higher Education Policy, 21, 439-457.

Esser, K., Hillebrand, W., Messner, D. y Meyer-Stamer, J. (1996). Competitividad sistémica: Nuevo desafío para las empresas y la política (New challenges for companies and politic). Revista de la Comisión Económica para América Latina (CEPAL), 59, 39-52.

Hazelkorn, E. (2008). Learning to live with league tables and ranking: The experience of institutional leaders. Higher Education Policy, 21, 193-216.

Heller, D. (2001). The States and Public Higher Education Policy: Affordability, Access, and Accountability. Baltimore, Ma: The Johns Hopkins University Press.

Hunt, S. (2000). A General Theory of Competition. London: Sage publications.

Kerr, C. (2007). The Gold and the Blue: Berkeley and Los Angeles, CA. University of California Press.

Kerr, C. (2001). The Uses of the University, Harvard University Press.

Lavados, J. (2006). Los Negocios Universitarios en el Mercado del Conocimiento, J.C. Sáez, editor.

Lemaitre, M.J. (2009). Nuevos enfoques sobre aseguramiento de la calidad en un contexto de cambios. Calidad en la Educación, CSE, n. ${ }^{\circ} 31$.

Marshall, J. (2010). Educación Superior: Institucionalidad para los nuevos desafíos. Calidad en la Educación, n. ${ }^{\circ} 32$.

McCaffery, P. (2010). The Higher Education Manager's Handbook. New York and London: Routledge.

Mohrman, K., Ma, W. y Baker, D. (2008). The Research university in transition: The emerging global model. Higher Education Policy, 21, 5-28. 
Muga, A. y Sotomayor, A. (2010). La actividad universitaria y sus perspectivas en relación con el desarrollo regional. Estudios Sociales, 118.

OECD (2007). Review of Tertiary Education in Chile.

Ortega y Gasset, J. (1930). Misión de la Universidad, Revista de Occidente, Alianza.

Reyes, C. (2011). Gobierno Universitario: Antiguos y nuevos desafíos para las universidades chilenas. Red Universitaria Cruz del Sur, Grupo de Estudios en Educación Superior, Año 1, n. ${ }^{\circ} 4$.

Rojas, C. (2012). Las universidades de "bien público" y su contribución al desarrollo. Una aproximación a partir de datos de Panel del CRUCH 2000-2010. Ponencia IV Encuentro SOCHER, Coquimbo, U. Católica del Norte, agosto.

Rojas, C. (2005). Mecesup 1999-2003: Una mirada a la economía política y sus resultados. Estudios Sociales, 115.

Rojas, C. (2001). Globalización y capacidades colectivas de aprendizaje tecnológico. Más Allá del Bosque, Ed. Dolmen.

Rodríguez, E. (2008). Información aseguramiento de la calidad en la educación superior. Reformas de la Educación Superior, Ed. Universidad Diego Portales.

Shattock, M. (2006). Managing Good Governance in Higher Education. OUP, Maidenhead.

Sporn, B. (2009). Gobierno y administración: tendencias estructurales y organizacionales. Desafíos y Perspectivas de la Dirección Estratégica de las Instituciones Universitarias, Arata, A. y Rodríguez, E. (eds.) Comisión Nacional de Acreditación, pp. 215-243.

Teichler, U. (2008). Diversification, trends and explanations of the shape and size of higher education. Higher Education, 56, 349-379.

Trow, M. (2000). From Mass Higher Education Access: The American Advantage. UC Berkeley, CSHE paper series.

World Economic Forum (2011). Global Competitiveness Report 2010-2011. Geneva, Switzerland. 